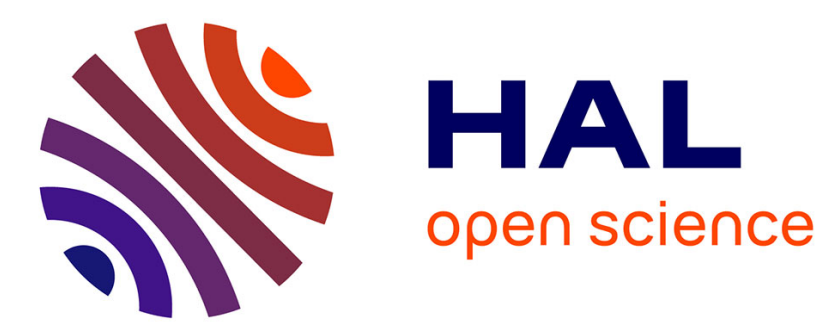

\title{
Closure of the Set of Diffusion Functionals - the One Dimensional Case
}

Jean-Jacques Alibert, Pierre Seppecher

\section{To cite this version:}

Jean-Jacques Alibert, Pierre Seppecher. Closure of the Set of Diffusion Functionals - the One Dimensional Case. Potential Analysis, 2008, 28 (4), pp.335-356. hal-00527074

\section{HAL Id: hal-00527074 \\ https://hal.science/hal-00527074}

Submitted on 18 Oct 2010

HAL is a multi-disciplinary open access archive for the deposit and dissemination of scientific research documents, whether they are published or not. The documents may come from teaching and research institutions in France or abroad, or from public or private research centers.
L'archive ouverte pluridisciplinaire HAL, est destinée au dépôt et à la diffusion de documents scientifiques de niveau recherche, publiés ou non, émanant des établissements d'enseignement et de recherche français ou étrangers, des laboratoires publics ou privés. 


\title{
Closure of the Set of Diffusion Functionals - the One Dimensional Case
}

\section{J. J. Alibert • P. Seppecher}

Received: 29 June 2005 / Accepted: 9 April 2007 /

Published online: 27 March 2008

(C) Springer Science + Business Media B.V. 2008

\begin{abstract}
We characterize the closure with respect to Mosco or $\Gamma$-convergence of the set of diffusion functionals in the one dimension case. As commonly accepted we find this closure is a set of local Dirichlet forms. The difficulty is to identify the right notion of locality. We compare different possible definitions. We give a representation theorem for the elements of the considered closure.
\end{abstract}

Keywords Homogenization $\cdot$ Mosco-convergence $\cdot \Gamma$-convergence $\cdot$ Local functionals • Dirichlet forms

Mathematics Subject Classifications (2000) $49 \mathrm{~J} 45 \cdot 49 \mathrm{~N} 10 \cdot 35 \mathrm{~J} 25$

\section{Introduction}

We are interested in the characterization of the different limits which can be reached by a sequence of diffusion functionals, i.e. of functionals of the kind

$$
F_{\alpha}(u):=\int_{\Omega} \alpha(x)|\nabla u(x)|^{2} d x,
$$

where the positive diffusion coefficient $\alpha$ belongs, like its inverse, to $L^{\infty}(\Omega)$.

These functionals belong to the set of Dirichlet forms. As this set has been proved [16] to be closed for the $\Gamma$-convergence, it is the natural framework of our study.

In dimension greater than two, well-known examples $[14,15,18,19]$ given by homogenization theory show that the limit functional can be non isotropic: it is then described by a diffusion matrix $A(x)$ and the limit functional takes the form:

$$
\int_{\Omega} \nabla u(x) \cdot A(x) \cdot \nabla u(x) d x .
$$


It has been proved [17] that the limit of functionals Eq. 1.1 takes the form Eq. 1.2 when the sequence of diffusion coefficients $\left(\alpha_{n}\right)$ and their inverses $\left(\alpha_{n}^{-1}\right)$ are bounded by a fixed real $M$. This is still true under weaker assumptions [8] but not in the general case.

In dimension greater than three, examples have been given (cf. [2, 3, 8, 16]), [7, 10] in which non local interactions arise at the limit. These interactions are represented by a non-negative measure $\gamma$ on $\Omega \times \Omega$ and the limit functional $F$ contains the nonlocal term or jumping term:

$$
\int_{\Omega \times \Omega}(u(x)-u(y))^{2} \gamma(d x d y) .
$$

Other examples [17] have been given in which the limit functional contains a socalled killing term of the form

$$
\int_{\Omega}(u(x))^{2} v(d x),
$$

where $v$ is a non-negative measure on $\Omega$. Let us notice immediately that we only consider in this paper functionals which vanish on constant fields $c$, or in an equivalent way, which are invariant when adding a constant :

$$
\forall c \in \mathbb{R}, \quad F(u+c)=F(u) .
$$

We call "objective" the functionals which satisfy this property. Any limit of a sequence of such functionals will inherit this property and we do not have to consider killing terms.

Recently, it was proved $[9,11]$ that, in dimension greater than three, the closure of the set of diffusion functionals coincides with the set of all objective Dirichlet forms.

In dimension two the characterization of the closure is still an open problem: very recently some fundamental differences between the two dimension case and greater dimension cases has been pointed out [6].

In the one dimension case it is a fact commonly accepted that any limit remains local. The goal of this paper is to establish this fact rigorously. In the literature there are many different ways for defining locality but we need to introduce a new definition adapted to our purpose. At the end of this paper we discuss the relations between the different notions of locality.

The paper is organized as follows: in Section 2 we describe the framework of objective Dirichlet forms and of Mosco or $\Gamma$-convergence. We set precisely the closure problem. We also give a simple example which shows the necessity of dealing with non regular Dirichlet forms.

In Section 3 we propose a new definition of locality and we state our main results. First we give a representation theorem for any local Dirichlet form. Then our main result states that this locality property characterizes the closure of the set of diffusion functionals. The proofs of these two theorems are rather long. There are splitted in several parts. In Subsection 3.2 we establish some characterizing properties of local Dirichlet forms. Subsection 3.3 is devoted to the proof of the representation theorem : many steps are necessary, the longest one being the construction of the underlying measure. Subsection 3.4 is devoted to the proof of the closure theorem. 
The major difficulty lies in the density result. The previous representation theorem plays here a crucial role.

In Section 4 we discuss the different notions of locality which can be found in the literature and we compare them by considering examples. These examples are based on the existence of "essential partitions" of the interval $(0,1)$, that is, partitions such that the intersection of any open interval with any piece of the partition has a non vanishing Lebesgue measure. We show that all the considered notions of locality are indeed different (and also different from the one we propose). We also prove that none of them are closed for the Mosco-convergence.

\section{Notations}

As this paper is concerned only by the one dimension case, $\Omega$ denotes a bounded open interval $\Omega:=(0,1) \subset \mathbb{R}$ and $\mathrm{L}^{2}(\Omega)$ is the usual space of square integrable (class of) functions with respect to the Lebesgue measure on $\Omega$. Note that in this whole paper, unless differently specified, the considered measure is the Lebesgue measure. We recall that the support of $u \in \mathrm{L}^{2}(\Omega)$ is the smallest closed subset $\Sigma$ of $\Omega$ such that $u(x)=0$ for almost every $x \in \Omega \backslash \Sigma$.

Let us first, following [13] introduce the set of Dirichlet forms.

Definition 1 We call Dirichlet form any functional on $\mathrm{L}^{2}(\Omega)$ satisfying the following property

i) $F$ is non negative: it takes values in $[0,+\infty]$.

ii) $\quad F$ is quadratic: its domain $D(F):=\left\{u \in \mathrm{L}^{2}(\Omega): F(u)<+\infty\right\}$ is a linear subspace of $\mathrm{L}^{2}(\Omega)$ and there exists a positive semi-definite bi-linear form $B$ such that $F(u)=B(u, u)$ for every $u$ in $D(F)$.

iii) $F$ is lower semi-continuous: it satisfies, for any $u \in \mathrm{L}^{2}(\Omega)$ and any sequence $\left(u_{n}\right)$ converging to $u$ :

$$
\liminf _{n \rightarrow \infty} F\left(u_{n}\right) \geq F(u) .
$$

iv) $F$ is Markovian: it satisfies for any $u \in \mathrm{L}^{2}(\Omega)$

$$
F(\underline{\bar{u}}) \leq F(u),
$$

where $\underline{\underline{u}}$ denotes the truncated function $\underline{\underline{u}}:=\max (0, \min (1, u))$.

Remark 1 By proposition 11.9 of [12], Property (ii) is equivalent to the fact that, for any $u$ and $v$ in $\mathrm{L}^{2}(\Omega)$ and any $t \geq 0$,

$$
F(u) \geq 0, F(t u) \leq t^{2} F(u), F(u+v)+F(u-v) \leq 2 F(u)+2 F(v) .
$$

Remark 2 Note that Property (ii) ensures that the domain $D(F)$ is non empty. In the present definition of Dirichlet forms the domain $D(F)$ is not necessarily dense in $\mathrm{L}^{2}(\Omega)$. We emphasize that, unlike many authors (e.g. [5]), we do not assume this density property. Indeed, as we will see later (see Example 1), this property is not preserved when passing to the limit with respect to the considered convergence of functionals. 
Definition 2 We call objective Dirichlet form any functional satisfying in addition to properties (i)-(iv),

v) $F$ is objective: it satisfies $F(c)=0$ for any constant function $c$.

We denote $\mathcal{Q}$ the set of all objective Dirichlet forms.

Remark 3 Property (v) is equivalent to

$$
\forall u \in \mathrm{L}^{2}(\Omega), \forall c \in \mathbb{R}, \quad F(u+c)=F(u) .
$$

To check this equivalence, it is enough to remark that, when $F(u)$ is finite, the quantity $F(u+c)-F(u)-F(c)$ is linear in $c$ and lower-bounded by $-F(u)$.

Definition 3 We say that $F \in \mathcal{Q}$ is a diffusion form if there exists $\alpha \in \mathrm{L}^{\infty}\left(\Omega, \mathbb{R}^{+*}\right)$ with $\alpha^{-1} \in \mathrm{L}^{\infty}\left(\Omega, \mathbb{R}^{+*}\right)$, such that

$$
F(u)= \begin{cases}\int_{\Omega} \alpha(x)\left(u^{\prime}(x)\right)^{2} d x & \text { if } u \in H^{1}(\Omega, \mathbb{R}), \\ +\infty & \text { otherwise }\end{cases}
$$

We denote $\mathcal{D}$ the set of all diffusion forms.

Definition 4 A sequence $\left(F_{n}\right)$ in $\mathcal{Q}$ Mosco-converges to $F$ if and only if it satisfies the following two properties:

i) Lower-bound inequality: For any sequence $\left(u_{n}\right)$ converging weakly to some $u$ in $\mathrm{L}^{2}(\Omega)$, the following lower-bound inequality holds :

$$
\liminf _{n \rightarrow \infty} F_{n}\left(u_{n}\right) \geq F(u) .
$$

ii) Upper-bound inequality : For every $u$ in $\mathrm{L}^{2}(\Omega)$, there exists an approximating sequence $\left(u_{n}\right)$ converging to $u$ strongly in $\mathrm{L}^{2}(\Omega)$ such that

$$
\limsup _{n \rightarrow \infty} F_{n}\left(u_{n}\right) \leq F(u) \text {. }
$$

Definition 5 Let $\mathfrak{U}$ be a subset of $\mathcal{Q}$, we call Mosco-closure of $\mathfrak{U}$ and denote $\overline{\mathfrak{U}}$ the set of all possible Mosco-limits of all sequences in $\mathfrak{U}$.

Our goal is then to characterize $\overline{\mathcal{D}}$.

Remark 4 As the properties (i), (iii), (iv), (v) and also (ii) in the form Eq. 2.3 easily pass to the limit, the set $\mathcal{Q}$ is closed for the Mosco-convergence: $\overline{\mathcal{Q}}=\mathcal{Q}$. The set $\mathcal{Q}$ is actually a good framework for our problem.

Remark 5 Mosco-convergence in the $\mathrm{L}^{2}(\Omega)$ topology is clearly a stronger notion than $\Gamma$-convergence for the strong topology of $L^{2}(\Omega)$ (refer to [12] for definition and properties of $\Gamma$-convergence). Then the Mosco-closure of a set is contained in its $\Gamma$-closure, i.e. in the set of all $\Gamma$-limits of all sequences in $\mathfrak{U}$. However, the previous closure result remains true even if one uses the $\Gamma$-convergence in the strong topology of $\mathrm{L}^{2}(\Omega)[16]$. Therefore our results can be interpreted in terms of $\Gamma$-convergence for the strong topology of $\mathrm{L}^{2}(\Omega)$. 
Though we will deal essentially with non regular Dirichlet forms, it is not useless to introduce the set $\mathcal{Q}_{r}$ of regular Dirichlet forms: let $C_{0}(\Omega)$ (or $C_{0}^{1}(\Omega)$ ) denote the set of continuous (resp. continuously differentiable) functions with compact support in $\Omega$. The form $F$ is said to be regular if there exists a subset of $D(F) \cap C_{0}(\Omega)$ dense in $C_{0}(\Omega)$ for the uniform norm and in $D(F)$ for the norm $\sqrt{\|u\|_{\mathrm{L}^{2}(\Omega)}^{2}+F(u)}$. The DenyBeurling formula [4] states that any regular and objective Dirichlet form admits on $C_{0}^{1}(\Omega)$ the following representation (in which $\eta$ is a non negative Radon measure on $\Omega$ while $\gamma$ is a symmetric non-negative Radon measure on $\Omega \times \Omega$ which does not concentrate on the diagonal):

$$
F(u)=\int_{\Omega}\left(u^{\prime}(x)\right)^{2} \eta(d x)+\int_{\Omega \times \Omega}(u(x)-u(y))^{2} \gamma(d x d y) .
$$

One refers usually to the first term of this representation as the "diffusion term" or "local term" and to the second one as the "jump term" or "non-local term".

The set $\mathcal{D}$ of diffusion forms is clearly included in the set of regular forms. This is not the case for the researched closure $\overline{\mathcal{D}}$. To illustrate this fact let us consider the following simple example.

Example 1 Let us consider the sequence of diffusion functionals $\left(F_{n}\right)$ in $\mathcal{D}$ defined by

$$
F_{n}(u)= \begin{cases}\int_{\Omega} \alpha_{n}(x)\left(u^{\prime}(x)\right)^{2} d x & \text { if } u \in H^{1}(\Omega, \mathbb{R}) \\ +\infty & \text { otherwise }\end{cases}
$$

where

$$
\alpha_{n}(x)= \begin{cases}n^{-1} & \text { if } x \in\left(\frac{1}{2}, \frac{1}{2}+\frac{1}{n}\right) \\ n & \text { otherwise. }\end{cases}
$$

We let the reader check that this sequence Mosco-converges to the functional $F \in \mathcal{Q}$ defined by

$$
F(u)= \begin{cases}(a-b)^{2} & \text { if } u=a \in \mathbb{R}, \text { a.e.in }\left(0, \frac{1}{2}\right), u=b \in \mathbb{R}, \text { a.e. in }\left(\frac{1}{2}, 1\right), \\ +\infty & \text { otherwise. }\end{cases}
$$

In this example $D(F)$ is a subspace of $\mathrm{L}^{2}(\Omega)$ of dimension 2. It is not a dense subspace. The functional $F$ which belongs to $\overline{\mathcal{D}}$ is definitively not a regular Dirichlet form.

\section{Main Results}

\subsection{Statement of the Main Results}

Recall that $x \in \Omega$ is called a Lebesgue point of $u \in L_{\ell o c}^{1}(\Omega)$ if there is a real number, let us call it $\tilde{u}(x)$, such that

$$
\lim _{\varepsilon \rightarrow 0} \frac{1}{2 \varepsilon} \int_{x-\varepsilon}^{x+\varepsilon}|u(t)-\tilde{u}(x)| d t=0
$$


The Lebesgue points of $u$ are thus points where $u$ does not oscillate too much, in an average sense. As well known, almost every $x \in \Omega$ is a Lebesgue point of $u$.

Let us introduce a notion of locality which is suitable for characterizing the closure of the set of diffusion forms.

Definition 6 For any $F \in \mathcal{Q}$, any compact $K \subset \Omega$ and any open interval $I \subset \Omega$ we set

$$
\begin{aligned}
& F(u, K):=\inf \{F(v) ; v=u \text { a.e. on some open set } O \supset K\}, \\
& F(u, I):=\sup \{F(u, K) ; K \text { compact, } K \subset I\} .
\end{aligned}
$$

We say that a form $F \in \mathcal{Q}$ is local if and only if, for any $u \in \mathrm{L}^{2}(\Omega)$, there exists a Borel measure $\mu_{u}$ such that $\mu_{u}(I)=F(u, I)$, for any open interval $I \subset \Omega$ and such that for any open set $O \subset \Omega$ and any Lebesgue point $x$ of $u$,

$$
\mu_{u}(O)=\mu_{u}(O \backslash\{x\})
$$

The set of all such forms is denoted $\mathcal{L}$.

The following theorem gives an integral representation for such local forms.

Theorem 1 Let $F \in \mathcal{L}$. Then there exists a unique closed subset $S$ of $\Omega$ and a unique Radon measure $\mu$ on $\Omega \backslash S$ such that, for every $u$ in the domain of $F$, the derivative $u^{\prime}$ of $u$ in the sense of distributions in $\Omega \backslash S$ can be written $u^{\prime}=\left(\frac{d u^{\prime}}{d \mu}\right) d \mu$ with $\frac{d u^{\prime}}{d \mu}$ in $L_{\mu}^{2}$ and

$$
F(u)=\int_{\Omega \backslash S}\left(\frac{d u^{\prime}}{d \mu}\right)^{2} d \mu
$$

This representation theorem is a key point for the proof of our following main result.

Theorem 2 The closure $\overline{\mathcal{D}}$ of the set of diffusion functionals coincides with the set of local Dirichlet forms $\mathcal{L}$.

\subsection{Characterization of Local Forms}

The following proposition gives a criterion to decide whether a functional $F$ belongs to $\mathcal{L}$ or not. For each Lebesgue point $x$ of $u \in L_{\ell o c}^{1}(\Omega)$, define

$$
\bar{u}_{x}^{\ell}:=u 1_{(0, x)}+\tilde{u}(x) 1_{(x, 1)} \quad \text { and } \quad \bar{u}_{x}^{r}:=\tilde{u}(x) 1_{(0, x)}+u 1_{(x, 1)} .
$$

Proposition 1 A Dirichlet form $F \in \mathcal{Q}$ belongs to $\mathcal{L}$ if and only if

$$
F(u)=F\left(\bar{u}_{x}^{\ell}\right)+F\left(\bar{u}_{x}^{r}\right),
$$

for every $u \in \mathrm{L}^{2}(\Omega)$ and every Lebesgue point $x$ of $u$.

Proof Let $F \in \mathcal{L}, u \in \mathrm{L}^{2}(\Omega)$ and $x$ be a Lebesgue point of $u$. The functional $v \rightarrow$ $\mu_{v}((s, t))$ is quadratic and objective for every open interval $(s, t) \subset \Omega$ which implies 
that, if $v=c$ a.e. in $(s, t)$ for some constant $c$ then $\mu_{v}((s, t))=0$. Since $x$ is also a Lebesgue point for $\bar{u}_{x}^{\ell}$ and $\bar{u}_{x}^{r}$, we have

$$
\begin{aligned}
F(u) & =\mu_{u}(\Omega)=\mu_{u}(\Omega \backslash\{x\})=\mu_{u}((0, x))+\mu_{u}((x, 1)) \\
& =\mu_{\bar{u}_{x}^{\ell}}((0, x))+\mu_{\bar{u}_{x}^{r}}((x, 1))=\mu_{\bar{u}_{x}^{\ell}}(\Omega \backslash\{x\})+\mu_{\bar{u}_{x}^{r}}(\Omega \backslash\{x\}) \\
& =\mu_{\bar{u}_{x}^{\ell}}(\Omega)+\mu_{\bar{u}_{x}^{r}}(\Omega)=F\left(\bar{u}_{x}^{\ell}\right)+F\left(\bar{u}_{x}^{r}\right)
\end{aligned}
$$

Conversely, assume that $F \in \mathcal{Q}$ is such that Eq. 3.6 holds. Let $u \in \mathrm{L}^{2}(\Omega)$ and $x \in \Omega$ be a Lebesgue point of $u$. For each $\delta>0$, there are two Lebesgue points $a_{\delta} \in(0, \delta)$, $x_{\delta} \in(x-\delta, x)$ of $u$ such that

$$
\left|\tilde{u}\left(a_{\delta}\right)\right| \leq \frac{1}{\delta} \int_{0}^{\delta}|u(t)| d t \quad \text { and } \quad\left|\tilde{u}\left(x_{\delta}\right)-\tilde{u}(x)\right| \leq \frac{1}{\delta} \int_{x-\delta}^{x}|u(t)-\tilde{u}(x)| d t .
$$

This implies that there exist two sequences $\left(a_{n}\right),\left(x_{n}\right)$ of Lebesgue points of $u$ such that $\left(a_{n}\right)$ decreases to $0,\left(x_{n}\right)$ increases to $x, \lim _{n} a_{n}\left(\tilde{u}\left(a_{n}\right)\right)^{2}=0$ and $\lim _{n}\left|\tilde{u}\left(x_{n}\right)-\tilde{u}(x)\right|=0$. Then $\bar{u}_{x_{n}}^{\ell}-\bar{u}_{a_{n}}^{\ell}+\tilde{u}\left(a_{n}\right)=u$ a.e. in $\left(a_{n}, x_{n}\right)$ and $\bar{u}_{x_{n}}^{\ell}-\bar{u}_{a_{n}}^{\ell}+\tilde{u}\left(a_{n}\right)$ converges to $\bar{u}_{x}^{\ell}$ with respect to the $\mathrm{L}^{2}(\Omega)$ norm. Hence

$$
F(u,(0, x)) \geq \liminf _{n} F\left(\bar{u}_{x_{n}}^{\ell}-\bar{u}_{a_{n}}^{\ell}+\tilde{u}\left(a_{n}\right)\right) \geq F\left(\bar{u}_{x}^{\ell}\right) \geq F(u,(0, x)) .
$$

In the same way, one can prove that $F(u, \Omega)=F(u)$ and $F(u,(x, 1))=F\left(\bar{u}_{x}^{r}\right)$. Then for every Lebesgue points $x<y$ of $u$,

$$
F(u,(0, x))=F\left(\bar{u}_{x}^{\ell}\right), F(u,(x, y))=F\left(\bar{u}_{y}^{\ell}-\bar{u}_{x}^{\ell}\right), F(u,(y, 1))=F\left(\bar{u}_{y}^{r}\right) .
$$

Define the subset $S_{u}$ of $\Omega$ by

$$
\left.S_{u}:=\{x \in \Omega: 0 \leq s<x<t \leq 1 \Longrightarrow F(u,(s, t))=+\infty)\right\} .
$$

Let $I$ be any connected component of the open set $\Omega \backslash S_{u}$ and $x_{1}<\ldots<x_{n}$ be $n$ Lebesgue points of $u$ in $I$. By Eq. 3.8, we have

$$
F\left(u,\left(x_{1}, x_{n}\right)\right)=\sum_{i=1}^{n-1} F\left(u,\left(x_{i}, x_{i+1}\right)\right)
$$

Then choose a Lebesgue point $x$ of $u$ in $I$ and consider the non decreasing function $f_{I}$ from $I$ to $\mathbb{R}$ defined by $f_{I}(t):=F(u,(x, t))$ if $t \geq x, f_{I}(t):=-F(u,(t, x))$ if $t<x$. Thus $f_{I}(t)-f_{I}(s)=F(u,(s, t))$ for every Lebesgue points $s<t$ of $u$.

Let now $y \in I$ be any Lebesgue point of $u$ and let us choose two sequences $\left(s_{n}\right)$ and $\left(t_{n}\right)$ of Lebesgue points of $u$ such that $\left(s_{n}\right)$ increases to $y,\left(t_{n}\right)$ decreases to $y$, $\lim _{n}\left|\tilde{u}\left(s_{n}\right)-\tilde{u}(y)\right|=0$ and $\lim _{n}\left|\tilde{u}\left(t_{n}\right)-\tilde{u}(y)\right|=0$. Using Eq. 3.10 we obtain

$$
F\left(u,\left(s_{1}, y\right)\right)+F\left(u,\left(y, t_{1}\right)\right)=F\left(u,\left(s_{1}, s_{n}\right)\right)+F\left(u,\left(s_{n}, t_{n}\right)\right)+F\left(u,\left(t_{n}, t_{1}\right)\right)<+\infty .
$$

Since $\liminf _{n} F\left(u,\left(s_{1}, s_{n}\right)\right) \geq F\left(u,\left(s_{1}, y\right)\right), \liminf _{n} F\left(u,\left(t_{n}, t_{1}\right)\right) \geq F\left(u,\left(y, t_{1}\right)\right)$, we get $\lim _{n} F\left(u,\left(s_{n}, t_{n}\right)\right)=0$. Then $f_{I}$ has no jump at any Lebesgue point of $u$. This implies that there exists a unique Radon measure $\mu_{I}$ on $I$ such that for every Lebesgue points $s<t \in I$ of $u, \mu_{I}((s, t))=f_{I}(t)-f_{I}(s)=F(u,(s, t))$. Then for every $s<t$ (Lebesgue points or not) such that $[s, t] \subset I$ we have

$$
\mu_{I}((s, t))=F(u,(s, t)) .
$$


Define the measure $\mu_{u}$ on the $\sigma$-field of Borel sets $B \subset \Omega$ by

$$
\mu_{u}(B):= \begin{cases}+\infty & \text { if } B \cap S_{u} \neq \emptyset \\ \sum_{I} \mu_{I}(B \cap I) & \text { if } B \cap S_{u}=\varnothing\end{cases}
$$

where the sum is taken over all the connected components of $\Omega \backslash S_{u}$.

Now let us check that $\mu_{u}$ is suitable. If $(s, t) \cap S_{u} \neq \emptyset$ then $F(u,(s, t))=+\infty$ which implies $\mu_{u}((s, t))=F(u,(s, t))$. If $(s, t) \cap S_{u}=\emptyset$ then there exists a unique connected component $I$ of $\Omega \backslash S_{u}$ such that $(s, t) \subset I$ which implies (by Eq. 3.11) that $\mu_{u}((s, t))=$ $F(u,(s, t))$. Then for every $(s, t) \subset \Omega$, we have

$$
\mu_{u}((s, t))=F(u,(s, t)) .
$$

Let $x \in \Omega$ be a Lebesgue point of $u$. If $x \in \Omega \backslash S_{u}$ then $\mu_{u}(\{x\})=\mu_{I}(\{x\})=0$ where $I$ is the connected component of $\Omega \backslash S_{u}$ containing $x$. If $x \in S_{u}$ then $\mu_{u}(\{x\})=+\infty$ and $\mu_{u}(O)=+\infty$ for every open set $O \subset \Omega$ such that $x \in O$. Then $F \in \mathcal{L}$.

The next proposition shows that minimization of any $F$ in $\mathcal{L}$ can be achieved by monotone functions. We do not know if this property characterizes the set $\mathcal{L}$.

Proposition 2 If $F \in \mathcal{L}, u \in \mathrm{L}^{2}(\Omega)$ and $x<y$ are two Lebesgue points of $u$ then there exists $v \in \mathrm{L}^{2}(\Omega)$ satisfying $(i) v=u$ a.e. in $\Omega \backslash[x, y]$, (ii) $v$ is monotone in $(x, y)$, (iii) $v \in[\tilde{u}(x), \tilde{u}(y)]$ a.e. in $(x, y),($ iv) $F(v) \leq F(u)$.

Proof Without loss of generality, let us assume that $\tilde{u}(x) \leq \tilde{u}(y)$. By induction, we construct a sequence of subdivisions $\sigma^{n}: t_{0}^{n}=x<t_{1}^{n}<\cdots<t_{2^{n}}^{n}=y$ with a step size $s_{n}:=\sup _{i}\left|t_{i+1}^{n}-t_{i}^{n}\right|$ tending to zero and an associated sequence of functions ( $\left.u_{n}\right)$ satisfying (a) $u_{n}=u$ a.e. in $\Omega \backslash[x, y]$, (b) each $t_{i}^{n}$ is a Lebesgue point of $u_{n}$, (c) $\tilde{u}\left(t_{i}^{n}\right) \leq u_{n} \leq \tilde{u}\left(t_{i+1}^{n}\right)$ in $\left(t_{i}^{n}, t_{i+1}^{n}\right)$, (d) $F\left(u_{n}\right) \leq F(u)$.

For $n=0$ we consider the trivial subdivision $x<y$ and the associated function $u_{0}$ defined by $u_{0}=: \min (\tilde{u}(y), \max (u, \tilde{u}(x)))$ in $[x, y]$ and $u_{0}:=u$ in $\Omega \backslash[x, y]$. Proposition 1 and Markov property ensure that $F\left(u_{0}\right) \leq F(u)$.

Assume that $\left(\sigma^{n}, u_{n}\right)$ is suitable. We define $\sigma^{n+1}$ by setting $t_{2 i}^{n+1}:=t_{i}^{n}$ and by choosing for $t_{2 i+1}^{n+1}$ a Lebesgue point of $u_{n}$ in $\left(\frac{2}{3} t_{i}^{n}+\frac{1}{3} t_{i+1}^{n}, \frac{1}{3} t_{i}^{n}+\frac{2}{3} t_{i+1}^{n}\right)$. For such a couple $\left(\sigma^{n}, u_{n}\right)$ we denote

$$
\begin{aligned}
& \bar{u}_{n+1}:=u 1_{\Omega \backslash[x, y]}+\sum_{1}^{2^{n+1}} \tilde{u}_{n}\left(t_{i}^{n+1}\right) 1_{\left(t_{i-1}^{n+1}, t_{i}^{n+1}\right)} \\
& \underline{u}_{n+1}:=u 1_{\Omega \backslash[x, y]}+\sum_{1}^{2^{n+1}} \tilde{u}_{n}\left(t_{i-1}^{n+1}\right) 1_{\left(t_{i-1}^{n+1}, t_{i}^{n+1}\right)}
\end{aligned}
$$

Then we define $u_{n+1}:=\min \left(\bar{u}_{n+1}, \max \left(u, \underline{u}_{n+1}\right)\right)$.

Proposition 1 and Markov property ensure that $F\left(u_{n+1}\right) \leq F\left(u_{n}\right)$. On the other hand, $\underline{u}_{n}$ and $\bar{u}_{n}$ are non decreasing in $(x, y), \underline{u}_{n}, \bar{u}_{n}$ belongs to $[\tilde{u}(x), \tilde{u}(y)]$ a.e. in $(x, y)$. Moreover

$$
\underline{u}_{n} \leq \underline{u}_{n+1} \leq u_{n+1} \leq \bar{u}_{n+1} \leq \bar{u}_{n}
$$


a.e. in $\Omega$, and

$$
\left\|\bar{u}_{n+1}-\underline{u}_{n+1}\right\|_{L^{1}(\Omega)} \leq \frac{2}{3}\left\|\bar{u}_{n}-\underline{u}_{n}\right\|_{L^{1}(\Omega)} .
$$

Then $u_{n}$ converges to some $v \in \mathrm{L}^{2}(\Omega)$ with $v$ monotone in $(x, z)$. Moreover $v \in$ $[\tilde{u}(x), \tilde{u}(y)]$ a.e. in $(x, y), v=u$ a.e. in $\Omega \backslash[x, y]$ and $F(v) \leq \liminf _{n} F\left(u_{n}\right) \leq F(u)$.

The characterization of $\mathcal{L}$ given by proposition 1 enables us to state the following lower-semi-continuity result.

Proposition 3 Let $F \in \mathcal{L}$ and $\left(u_{n}\right)$ be a sequence in $\mathrm{L}^{2}(\Omega)$ strongly converging to some $u$. Then $\liminf _{n} F\left(u_{n},(s, t)\right) \geq F(u,(s, t))$ for every open interval $(s, t) \subset \Omega$.

Proof Without loss of generality, assume that $u_{n}$ converges to $u$ a.e. in $\Omega$ and $\liminf _{n} F\left(u_{n},(s, t)\right)=\lim _{n} F\left(u_{n},(s, t)\right)<+\infty$. Let $s^{\prime}<t^{\prime}$ two points in $(s, t)$. Choose $x \in\left(s, s^{\prime}\right)$ and $y \in\left(t^{\prime}, t\right)$ such that $x$ and $y$ are Lebesgue points of $u$ and of every $u_{n}$ and such that $\lim _{n} \tilde{u}_{n}(x)=\tilde{u}(x)$ and $\lim _{n} \tilde{u}(y)=\tilde{u}(y)$. We have

$$
\lim _{n} \|\left({\overline{\left(u_{n}\right)_{y}^{\ell}}}_{-}-{\overline{\left(u_{n}\right)_{x}^{\ell}}}^{\ell}-\left(\bar{u}_{y}^{\ell}-\bar{u}_{x}^{\ell}\right) \|_{\mathrm{L}^{2}(\Omega)}=0 .\right.
$$

Hence

$$
\liminf _{n} F\left(u_{n},(s, t)\right) \geq \liminf _{n} F\left(\overline{\left(u_{n}\right)_{y}^{\ell}}-\overline{\left(u_{n}\right)_{x}^{\ell}}\right) \geq F\left(\bar{u}_{y}^{\ell}-\bar{u}_{x}^{\ell}\right) \geq F\left(u,\left[s^{\prime}, t^{\prime}\right]\right) .
$$

The proposition is proved as the previous inequality holds for any $\left[s^{\prime}, t^{\prime}\right] \subset(s, t)$.

\subsection{Proof of the Representation Theorem}

\subsubsection{Construction of the Set $S$}

Let us introduce the set $S$ where, roughly speaking, the value of $F(u)$ does not provide any control on the variation of $u$.

Proposition 4 To any $F \in \mathcal{Q}$, we associate the set

$$
S:=\left\{x \in \Omega: F\left(1_{(x, 1)}\right)=0\right\} .
$$

If $F \in \mathcal{L}, u \in \mathrm{L}^{2}(\Omega)$, let $\mu_{u}$ be the measure associated to $F$ and $u$ by Definition 6 and $(s, t)$ an open interval of $\Omega$ such that $\mu_{u}(s, t)<+\infty$. Then

$$
\mu_{u}((s, t) \cap S)=0 .
$$

Remark 6 Clearly $\Omega \backslash S$ is an open set. Indeed, if $\left(x_{n}\right)$ is a sequence in $S$ converging to some $x \in \Omega$, we have $\lim _{n}\left\|1_{\left(x_{n}, 1\right)}-1_{(x, 1)}\right\|_{\mathrm{L}^{2}(\Omega)}=0$. The lower semi-continuity of $F$ implies $F\left(1_{(x, 1)}\right) \leq \liminf _{n} F\left(1_{\left(x_{n}, 1\right)}\right) \leq 0$. Thus $x$ belongs to $S$. Proposition 4 shows that energy never concentrates on $S$.

Proof of Proposition 4 Let $x \in S$. For every $\alpha, \beta \in R$ we have

$$
F\left(\alpha 1_{(0, x)}+\beta 1_{(x, 1)}\right)=(\beta-\alpha)^{2} F\left(1_{(x, 1)}\right)=0
$$


Let $x \in S \cap(s, t)$. Let $\left(s_{n}\right)$ and $\left(t_{n}\right)$ be two sequences in $(s, t)$ of Lebesgue points of $u$ such that $x-\frac{1}{n}<s_{n}<x<t_{n}<x+\frac{1}{n}$ and

$$
\left|\tilde{u}\left(s_{n}\right)\right| \leq n \int_{x-\frac{1}{n}}^{x}|u(t)| d t \quad \text { and } \quad\left|\tilde{u}\left(t_{n}\right)\right| \leq n \int_{x}^{x+\frac{1}{n}}|u(t)| d t
$$

Jensen's inequality implies that $\lim _{n}\left(x-s_{n}\right)\left|\tilde{u}\left(s_{n}\right)\right|^{2}=0=\lim _{n}\left(t_{n}-x\right)\left|\tilde{u}\left(t_{n}\right)\right|^{2}$. Define $u_{n}:=u 1_{\left(0, s_{n}\right)}+\tilde{u}\left(s_{n}\right) 1_{\left(s_{n}, x\right)}+\tilde{u}\left(t_{n}\right) 1_{\left(x, t_{n}\right)}+u 1_{\left(t_{n}, b\right)}$.

Since $\lim _{n}\left\|u-u_{n}\right\|_{L 2(\Omega)}=0$ we deduce from Proposition 3 that

$$
\begin{aligned}
\mu_{u}((s, t) \backslash\{x\}) & =\lim _{n} \mu_{u}\left(\left(s, s_{n}\right)\right)+\mu_{u}\left(\left(t_{n}, t\right)\right) \\
& =\lim _{n} F\left(u,\left(s, s_{n}\right)\right)+F\left(u,\left(t_{n}, t\right)\right) \\
& =\lim _{n} F\left(u_{n},(s, t)\right) \\
& \geq F(u,(s, t)) \geq \mu_{u}((s, t))
\end{aligned}
$$

Since $\mu_{u}((s, t))<+\infty$ we conclude that

$$
\forall x \in S \cap(s, t) \quad \mu_{u}(\{x\})=0 .
$$

Let $S_{\sigma}$ be the set of these $x \in S$ such that $S \cap x-\varepsilon, x+\varepsilon[=\{x\}$ for some $\varepsilon>0$. Since $S_{\sigma}$ is at most countable, we deduce from Eq. 3.16 that

$$
\mu_{u}\left((s, t) \cap S_{\sigma}\right)=0 .
$$

Let $h>0$ and consider a subdivision $s=t_{0}<\ldots<t_{n+1}=t$ of $(s, t)$ such that $\mid t_{i}-$ $t_{i-1} \mid<h$ and $t_{1}, \ldots, t_{n}$ are Lebesgue points of $u$. Denote $\left.I_{i}:=\right] t_{i}, t_{i+1}[$ and let $J$ be the set of these $i \in\{1, \ldots, n-1\}$ such that $I_{i} \cap\left(S \backslash S_{\sigma}\right) \neq \emptyset$. If $i \in J$ then $I_{i}$ contains at least two points $s_{i}^{1}<s_{i}^{2}$ of $S$. Define $v_{h} \in \mathrm{L}^{2}(\Omega)$ by

$$
v_{h}(x):= \begin{cases}\tilde{u}\left(t_{i}\right) & \text { if } x \in\left(t_{i}, s_{i}^{1}\right) \\ c_{i} & \text { if } x \in\left[s_{i}^{1}, s_{i}^{2}\right] \\ \tilde{u}\left(t_{i+1}\right) & \text { if } x \in\left(s_{i}^{2}, t_{i+1}\right),\end{cases}
$$

if $x \in I_{i}$ for some $i \in J, v_{h}(x):=u(x)$ otherwise. Here $c_{i}$ is a real tuned in such a way that $\int_{I_{i}} u(t) d t=\int_{I_{i}} v_{h}(t) d t$.

As $h$ tends to zero, the sequence $\left(v_{h}\right)$ converges to $u$ in $\mathrm{L}^{2}(\Omega)$ then, by Proposition 3, $\liminf \operatorname{in}_{h \downarrow} F\left(v_{h},(s, t)\right) \geq F(u,(s, t))$. On the other hand, using Eq. 3.15 we have, for any $i \in J, F\left(v_{h}, I_{i}\right)=0$. As, for any $i \notin J, F\left(v_{h}, I_{i}\right)=F\left(u, I_{i}\right)$, we have

$$
\begin{aligned}
\mu_{u}\left((s, t) \cap\left(S \backslash S_{\sigma}\right)\right) & \leq F\left(u, I_{0}\right)+F\left(u, I_{n}\right)+\sum_{i \in J} F\left(u, I_{i}\right) \\
& \leq F(u,(s, t))-F\left(v_{h},(s, t)\right)+F\left(u, I_{0}\right)+F\left(u, I_{n}\right) .
\end{aligned}
$$

Passing to the limsup as $h$ tends to zero, we get

$$
\mu_{u}\left((s, t) \cap\left(S \backslash S_{\sigma}\right)\right) \leq 0 .
$$

Proof of Proposition 4 is completed recalling Eq. 3.17. 


\subsubsection{Construction of the Measure $\mu$}

Proposition 5 Let $s \leq t$ in $\Omega$. Let $A(s, t)$ be the set of these $u \in \mathrm{L}^{2}(\Omega)$ such that $u \leq 0$ a.e. in $\Omega \cap(0, s)$ and $u \geq 1$ a.e. in $\Omega \cap(t, 1)$. For each $F$ in $\mathcal{Q}$, define

$$
m(s, t):=\inf \{F(u): u \in A(s, t)\} .
$$

If $F \in \mathcal{L}$ then there exists a unique Radon measure $\mu$ on $\Omega \backslash S$ such that

$$
\mu([s, t])=\frac{1}{m(s, t)}
$$

for every closed interval $[s, t] \subset \Omega \backslash S$.

We need to state several properties for $m(s, t)$ before proving Proposition 5.

Lemma 1 Let $F \in \mathcal{L}$. Then

i) $S:=\{x \in \Omega: \forall s, t \in \Omega(s<x<t \Longrightarrow m(s, t)=0)\}$.

ii) Let $x \in \Omega$, for every sequences $\left(s_{n}\right)$ in $(0, x)$ and $\left(t_{n}\right)$ in $(x, 1)$ converging to $x$,

$$
\lim _{n} m\left(s_{n}, t_{n}\right)=m(x, x) .
$$

iii) If $[s, t] \subset \Omega \backslash S$ then $m(s, t)>0$.

Proof Point (i) is obvious. For each integer $n$, owing to the properties of Dirichlet forms, there exists a minimizer $u_{n} \in A\left(s_{n}, t_{n}\right)$ such that $u_{n}=0$ a.e. in $\left(0, s_{n}\right), u_{n}=1$ a.e. in $\left(t_{n}, 1\right)$. and $F\left(u_{n}\right)=m\left(s_{n}, t_{n}\right)$. Since $\lim _{n}\left\|u_{n}-1_{(x, 1)}\right\|_{\mathrm{L}^{2}(\Omega)}=0$ we have

$$
\begin{aligned}
m(x, x) & \geq \limsup _{n} m\left(s_{n}, t_{n}\right) \geq \liminf _{n} m\left(s_{n}, t_{n}\right) \\
& \geq \liminf _{n} F\left(u_{n}\right) \geq F\left(1_{(x, 1)}\right) \geq m(x, x) .
\end{aligned}
$$

Assume that $[s, t] \subset \Omega \backslash S$ and $m(s, t)=0$. By Proposition 2 there is a non decreasing $u \in A(s, t)$ such that $F(u)=0$. Owing to (i), for each $x \in[s, t]$ there exist Lebesgue points of $u$ such that $s^{\prime}<x<t^{\prime}$ et $m\left(s^{\prime}, t^{\prime}\right)>0$. Since $F(u) \geq\left(\tilde{u}\left(t^{\prime}\right)-\tilde{u}\left(s^{\prime}\right)\right)^{2} m\left(s^{\prime}, t^{\prime}\right)$, $\tilde{u}\left(t^{\prime}\right)=\tilde{u}\left(s^{\prime}\right)$. Since $u$ is non decreasing, $u$ is constant in a neighborhood of $[s, t]$ which contradicts $u \in A(s, t)$.

Lemma 2 Let $F \in \mathcal{L}$ and I be a connected component of $\Omega \backslash S$. If $s \leq s^{\prime}<t^{\prime} \leq t \in I$ then

$$
\frac{1}{m(s, t)} \geq \frac{1}{m\left(s, s^{\prime}\right)}+\frac{1}{m\left(t^{\prime}, t\right)}
$$

Proof There exist $u \in A\left(s, s^{\prime}\right)$ and $v \in A\left(t, t^{\prime}\right)$ satisfying : $u=0$ a.e. in $(0, s), v=0$ a.e. in $\left(0, t^{\prime}\right), u=1$ a.e. in $\left(s^{\prime}, 1\right), v=1$ a.e. in $(t, 1), F(u)=m\left(s, s^{\prime}\right)$ and $F(v)=m\left(t^{\prime}, t\right)$. Any $x \in\left(s^{\prime}, t^{\prime}\right)$ is a Lebesgue point of the function $w \in A(s, t)$ defined by

$$
w:=\left(\frac{F(v)}{F(u)+F(v)}\right) u+\left(\frac{F(u)}{F(u)+F(v)}\right) v
$$


Then $m(s, t) \leq F(w)$ and by Proposition 1 ,

$$
F(w)=F\left(\bar{w}_{x}^{\ell}\right)+F\left(\bar{w}_{x}^{r}\right)=\frac{F(u) F(v)}{F(u)+F(v)}=\frac{m\left(s, s^{\prime}\right) m\left(t^{\prime}, t\right)}{m\left(s, s^{\prime}\right)+m\left(t^{\prime}, t\right)},
$$

which leads to Eq. 3.21.

Lemma 3 If $F \in \mathcal{L}$ then the set $\{x \in \Omega \backslash S: m(x, x)<+\infty\}$ is at most countable.

Proof Let $I$ be a connected component of $\Omega \backslash S, n$ be a positive integer and $s<t$ in $I$. If $x_{1}, \ldots, x_{p}$ are $p$ elements of the set $\{x \in[s, t]: m(x, x) \leq n\}$ then Lemma 2 give us the estimate

$$
p \leq \sum_{i=1}^{p} \frac{n}{m\left(x_{i}, x_{i}\right)} \leq \frac{n}{m(s, t)} .
$$

Thus $\{x \in[s, t]: m(x, x) \leq n\}$ is a finite set.

Lemma 4 Let $F \in \mathcal{L}$ and I be a connected component of $\Omega \backslash S$. Then

i) for every $s<t$ in $I$, there exists a Lebesgue measurable set $I_{(s, t)}^{*} \subset(s, t)$ such that $\left|(s, t) \backslash I_{(s, t)}^{*}\right|=0, m(x, x)=+\infty$ for every $x \in I_{(s, t)}^{*}$ and

$$
\frac{1}{m(s, t)}=\frac{1}{m\left(s, x_{1}\right)}+\frac{1}{m\left(x_{n}, t\right)}+\sum_{i=1}^{n-1} \frac{1}{m\left(x_{i}, x_{i+1}\right)}
$$

for every $x_{1}<\ldots<x_{n}$ belonging to $I_{(s, t)}^{*}$.

ii) there exists a Lebesgue measurable set $I^{*} \subset I$ such that $\left|I \backslash I^{*}\right|=0, m(x, x)=$ $+\infty$ for every $x \in I^{*}$ and

$$
\frac{1}{m\left(x_{1}, x_{n}\right)}=\sum_{i=1}^{n-1} \frac{1}{m\left(x_{i}, x_{i+1}\right)}
$$

for every $x_{1}<\ldots<x_{n}$ belonging to $I^{*}$.

Proof Let $s<t$ in $I$ and $w \in A(s, t)$ be such that $F(w)=m(s, t)$. Define

$$
I_{(s, t)}^{*}:=\{x \in(s, t): m(x, x)=+\infty, x \text { is a Lebesgue point of } w\} .
$$

By Lemma $3\left|(s, t) \backslash I_{(s, t)}^{*}\right|=0$. Let $x_{1}, \ldots, x_{n} \in I_{(s, t)}^{*}$ be such that $x_{1}<\ldots<x_{n}$. Denote $x_{0}=s, x_{n+1}:=t, m_{i}:=m\left(x_{i-1}, x_{i}\right)$ if $1 \leq i \leq n+1, \alpha_{1}:=\tilde{w}\left(x_{1}\right), \alpha_{n+1}:=1-\tilde{w}\left(x_{n}\right)$ and $\alpha_{i}:=\tilde{w}\left(x_{i}\right)-\tilde{w}\left(x_{i-1}\right)$, if $2 \leq i \leq n$. Using Proposition 1 , identity $\sum_{i=1}^{n+1} \alpha_{i}=1$ and Cauchy-Schwarz inequality, we obtain

$$
F(w)=F\left(\bar{w}_{x_{1}}^{\ell}\right)+F\left(\bar{w}_{x_{n}}^{r}\right)+\sum_{i=2}^{n} F\left(\bar{w}_{x_{i}}^{\ell}-\bar{w}_{x_{i-1}}^{\ell}\right) \geq \sum_{i=1}^{n+1}\left(\alpha_{i}\right)^{2} m_{i} \geq\left(\sum_{i=1}^{n+1} \frac{1}{m_{i}}\right)^{-1},
$$

which leads to

$$
\frac{1}{m(s, t)} \leq \sum_{i=1}^{n+1} \frac{1}{m\left(x_{i-1}, x_{i}\right)}
$$


For each $i \in\{1, \ldots, n\}$, there is $w_{i} \in A\left(x_{i-1}, x_{i}\right)$ such that $F\left(w_{i}\right)=m\left(x_{i-1}, x_{i}\right)$. Let $\left(x_{i}^{(p)}\right)$ be a sequence of Lebesgue points of $w_{i}$ increasing to $x_{i}$. By Proposition 1 we have

$$
\begin{aligned}
m\left(x_{i-1}, x_{i}\right) & \left.=F\left(\overline{\left(w_{i}\right)_{x_{i}^{(p)}}^{\ell}}\right)+F\left(\overline{\left(w_{i}\right.}\right)_{x_{i}^{(p)}}^{r}\right) \\
& \geq\left(\tilde{w}_{i}\left(x_{i}^{(p)}\right)\right)^{2} m\left(x_{i-1}, x_{i}^{(p)}\right)+\left(1-\tilde{w}_{i}\left(x_{i}^{(p)}\right)\right)^{2} m\left(x_{i}^{(p)}, x_{i}\right) \\
& \geq \frac{m\left(x_{i-1}, x_{i}^{(p)}\right) m\left(x_{i}^{(p)}, x_{i}\right)}{m\left(x_{i-1}, x_{i}^{(p)}\right)+m\left(x_{i}^{(p)}, x_{i}\right)} .
\end{aligned}
$$

Using Lemma 2 we obtain

$$
\begin{aligned}
\sum_{i=1}^{n+1} \frac{1}{m\left(x_{i-1}, x_{i}\right)} & =\frac{1}{m\left(x_{n}, x_{n+1}\right)}+\sum_{i=1}^{n} \frac{1}{m\left(x_{i-1}, x_{i}\right)} \\
& \leq \frac{1}{m\left(x_{n}, x_{n+1}\right)}+\sum_{i=1}^{n} \frac{1}{m\left(x_{i-1}, x_{i}^{(p)}\right)}+\sum_{i=1}^{n} \frac{1}{m\left(x_{i}^{(p)}, x_{i}\right)} \\
& \leq \frac{1}{m(s, t)}+\sum_{i=1}^{n} \frac{1}{m\left(x_{i}^{(p)}, x_{i}\right)}
\end{aligned}
$$

Using Lemma 1 and passing to the limit as $p$ tends to $\infty$ in the above inequality, we obtain

$$
\frac{1}{m(s, t)} \geq \sum_{i=1}^{n+1} \frac{1}{m\left(x_{i-1}, x_{i}\right)}
$$

which together with Eq. 3.24 leads to Eq. 3.22.

Since we also have

$$
\frac{1}{m\left(s, x_{1}\right)}+\frac{1}{m\left(x_{1}, x_{n}\right)}+\frac{1}{m\left(x_{n}, t\right)}=\frac{1}{m(s, t)}
$$

we obtain Eq. 3.23. The proof of Lemma 4 is completed by using sequences $\left(s_{q}\right),\left(t_{q}\right)$ such that $\left(s_{q}\right)$ decreases, $\left(t_{q}\right)$ increases, $I:=\bigcup_{q}\left(s_{q}, t_{q}\right)$ and defining $I^{*}:=\bigcap_{q} I_{\left(s_{q}, t_{q}\right)}^{*}$.

Proof of Proposition 5 Let $I$ be any connected component of the open set $\Omega \backslash S$ and $I^{*}$ be a Lebesgue measurable set as in point (ii) of Lemma 4. Choose $x \in I^{*}$ and define $f_{I}$ from $I$ to $\mathbb{R}$ by

$$
f_{I}(t):= \begin{cases}m(x, t)^{-1} & \text { if } t>x, \\ 0 & \text { if } t=x, \\ -m(t, x)^{-1} & \text { if } t<x .\end{cases}
$$

Clearly $f_{I}$ is non decreasing, then there exists a unique Radon measure $\mu_{I}$ on $I$ such that $f_{I}\left(t^{+}\right)-f_{I}\left(s^{-}\right)=\mu_{I}([s, t])$ for every $s<t$ in $I$. By Lemma $1, f_{I}$ has no jump at any point of $I^{*}$ then Lemma 4 implies that $\mu_{I}([s, t])=m(s, t)^{-1}$ if $s, t$ in $I^{*}$. Let $x \in I$ and choose two sequences $\left(s_{n}\right)$ and $\left(t_{n}\right)$ in $I^{*}$ such that $\left(s_{n}\right)$ increases to $x,\left(t_{n}\right)$ decreases to $x$. By Lemma 1

$$
\mu_{I}(\{x\})=\lim _{n} \mu_{I}\left(\left[s_{n}, t_{n}\right]\right)=\lim _{n} m\left(s_{n}, t_{n}\right)^{-1}=m(x, x)^{-1}
$$


Let $s<t$ in $I$ and $I_{(s, t)}^{*}$ be a Lebesgue measurable set as in part (i) of Lemma 4. Choose two sequences $\left(s_{n}\right)$ and $\left(t_{n}\right)$ in $I_{(s, t)}^{*} \cap I^{*}$ such that $\left(s_{n}\right)$ decreases to $s,\left(t_{n}\right)$ increases to $t$ and $s_{n}<t_{n}$. We have

$$
\begin{aligned}
m(s, t)^{-1} & =\lim _{n} m\left(s, s_{n}\right)^{-1}+m\left(s_{n}, t_{n}\right)^{-1}+m\left(t_{n}, t\right)^{-1} \\
& =\lim _{n} m\left(s, s_{n}\right)^{-1}+\mu_{I}\left(\left[s_{n}, t_{n}\right]\right)+m\left(t_{n}, t\right)^{-1} \\
& =m(s, s)^{-1}+\mu_{I}((s, t))+m(t, t)^{-1} \\
& =\mu_{I}(\{s\})+\mu_{I}((s, t))+\mu_{I}(\{t\}) \\
& =\mu_{I}([s, t])
\end{aligned}
$$

In order to complete the proof of Proposition 5, we define the measure $\mu$ on the $\sigma$-field of Borel sets $B \subset \Omega \backslash S$ by

$$
\mu(B):=\sum_{I} \mu_{I}(B \cap I) .
$$

where the sum is taken over all the connected components of $\Omega \backslash S$.

\subsubsection{Proof of Theorem 1}

Lemma 5 Let $F \in \mathcal{L}, u \in \mathrm{L}^{2}(\Omega)$ and $(s, t)$ be an open interval of $\Omega$. If $F(u,(s, t))<$ $+\infty$ then $u \in B V_{\ell o c}((s, t) \backslash S)$ and there exists $f \in L_{\mu}^{2}((s, t) \backslash S)$ such that

$$
\int_{\Omega \backslash S} u(x) \varphi^{\prime}(x) d x=-\int_{\Omega \backslash S} \varphi f d \mu
$$

for every $\varphi \in C_{c}^{\infty}((s, t) \backslash S)$.

Proof Let $I$ be a connected component of $\Omega \backslash S$ and $h>0$ be such that $[x-h, x+$ $h] \subset(s, t) \backslash S$ for every $x$ belonging to the compact support of $\varphi$. There are $x_{0}<x_{1}<$ $\ldots<x_{n+1}$ in $I \cap(s, t)$ such that $h=x_{i+1}-x_{i}$ and $\left(x_{0}, x_{n+1}\right) \supset I \cap \operatorname{support} \varphi$. Denoting $U_{i}=h^{-1} \int_{x_{i-1}}^{x_{i}} u(t) d t$ we have

$$
\begin{aligned}
\int_{I} \varphi^{\prime}(x) u(x) d x & =\varepsilon(h)+\sum_{i=1}^{n+1}\left(\varphi\left(x_{i}\right)-\varphi\left(x_{i-1}\right)\right) U_{i} \\
& =\varepsilon(h)-\sum_{i \text { even }} \varphi\left(x_{i}\right)\left(U_{i+1}-U_{i}\right)-\sum_{i \text { odd }} \varphi\left(x_{i}\right)\left(U_{i+1}-U_{i}\right)
\end{aligned}
$$

For each even $i \in[1, n]$ choose $t_{i} \in\left(x_{i-1}, x_{i}\right)$ and $t_{i+1} \in\left(x_{i}, x_{i+1}\right)$ which are Lebesgue points of $u, \mu\left(\left\{t_{i}\right\}\right)=\mu\left(\left\{t_{i+1}\right\}\right)=0$ and $\left(\tilde{u}\left(t_{i+1}\right)-\tilde{u}\left(t_{i}\right)\right)^{2} \geq\left(U_{i+1}-U_{i}\right)^{2}$. For each odd $i \in[1, n]$ choose $s_{i} \in\left(x_{i-1}, x_{i}\right)$ and $s_{i+1} \in\left(x_{i}, x_{i+1}\right)$ which are Lebesgue points of $u$, 
$\mu\left(\left\{s_{i}\right\}\right)=\mu\left(\left\{s_{i+1}\right\}\right)=0$ and $\left(\tilde{u}\left(s_{i+1}\right)-\tilde{u}\left(s_{i}\right)\right)^{2} \geq\left(U_{i+1}-U_{i}\right)^{2}$. Using Cauchy-Schwarz inequality we obtain

$$
\begin{aligned}
\left(\sum_{i \text { even }} \varphi\left(x_{i}\right)\left(U_{i+1}-U_{i}\right)\right)^{2} \leq & \sum_{i \text { even }} \varphi\left(x_{i}\right)^{2} \mu(] t_{i}, t_{i+1}[) \\
& \times \sum_{i \text { even }}\left(\tilde{u}\left(t_{i+1}\right)-\tilde{u}\left(t_{i}\right)\right)^{2} m\left(t_{i}, t_{i+1}\right) .
\end{aligned}
$$

then

$$
\left(\sum_{i \text { even }} \varphi\left(x_{i}\right)\left(U_{i+1}-U_{i}\right)\right)^{2} \leq F(u,(s, t)) \sum_{i \text { even }} \varphi\left(x_{i}\right)^{2} \mu(] t_{i}, t_{i+1}[) .
$$

Similar estimate holds for odd $i$. Then passing to the limit in Eq. 3.26 as $h \downarrow 0$ leads to

$$
\left|\int_{I} \varphi^{\prime}(x) u(x) d x\right|^{2} \leq 2 F(u,(s, t))\|\varphi\|_{L_{\mu}^{2}(I)}^{2},
$$

which is enough to complete the proof.

Lemma 6 Let $F \in \mathcal{L}, u \in \mathrm{L}^{2}(\Omega)$ and $(s, t)$ be an open interval of $\Omega$. If $F(u,(s, t))<$ $+\infty$ then

$$
F(u,(s, t)) \geq \int_{(s, t) \backslash S}\left(\frac{d u^{\prime}}{d \mu}\right)^{2} d \mu
$$

where $\frac{d u^{\prime}}{d \mu}$ denotes the derivative of $u^{\prime}$ in the sense of Radon-Nikodym in $(s, t) \backslash S$.

Proof By Lemma 5, $u \in B V_{\ell o c}((s, t) \backslash S)$. Let $[x, y] \subset(s, t) \backslash S$ such that $\mu(\{x\})=$ $\mu(\{y\})=0$. This implies that $x$ and $y$ are Lebesgue points of $u$. Then we have

$$
\tilde{u}(y)-\tilde{u}(x)=\int_{(x, y)} \frac{d u^{\prime}}{d \mu} d \mu .
$$

Let $I$ be a connected component of $\Omega \backslash S, \varphi \in C_{c}^{\infty}((s, t) \backslash S)$ and $x_{0}<x_{1}<\ldots<$ $x_{n}$ such that $I \cap \operatorname{support}(\varphi) \subset\left[x_{0}, x_{n}\right] \subset I \cap(s, t)$, and $\mu\left(\left\{x_{i}\right\}\right)=0$. Denoting $h=$ $\max _{i}\left\{x_{i}-x_{i-1}\right\}$ and choosing points $s_{i} \in\left(x_{i-1}, x_{i}\right)$ we have

$$
\int_{I} \varphi \frac{d u^{\prime}}{d \mu} d \mu=\sum_{i=1}^{n} \int_{\left(x_{i-1}, x_{i}\right)} \varphi \frac{d u^{\prime}}{d \mu} d \mu=\varepsilon(h)+\sum_{i=1}^{n} \varphi\left(s_{i}\right)\left(\tilde{u}\left(x_{i}\right)-\tilde{u}\left(x_{i-1}\right)\right) .
$$

Using Cauchy-Schwarz inequality we obtain

$$
\begin{aligned}
\left(\int_{I} \varphi \frac{d u^{\prime}}{d \mu} d \mu+\varepsilon(h)\right)^{2} \leq & \sum_{i=1}^{n} \varphi\left(s_{i}\right)^{2} \mu\left(\left[x_{i-1}, x_{i}\right]\right) \\
& \times \sum_{i=1}^{n}\left(\tilde{u}\left(x_{i}\right)-\tilde{u}\left(x_{i-1}\right)\right)^{2} m\left(x_{i-1}, x_{i}\right) \\
\leq & F(u,(s, t)) \sum_{i=1}^{n}\left(\varphi\left(s_{i}\right)\right)^{2} \mu\left(\left[x_{i-1}, x_{i}\right]\right) .
\end{aligned}
$$


Then passing to the limit as $h$ tends to 0 leads to

$$
\left|\int_{I} \varphi \frac{d u^{\prime}}{d \mu} d \mu\right|^{2} \leq F(u,(s, t))\|\varphi\|_{L_{\mu}^{2}(I)}^{2} .
$$

which is enough to complete the proof.

Lemma 7 Let $F \in \mathcal{L}, u \in \mathrm{L}^{2}(\Omega)$ and $(s, t)$ be an open interval of $\Omega$. If $F(u,(s, t))<$ $+\infty$ then

$$
F(u,(s, t)) \leq \int_{(s, t) \backslash S}\left(\frac{d u^{\prime}}{d \mu}\right)^{2} d \mu
$$

where $\frac{d u^{\prime}}{d \mu}$ denotes the derivative of $u^{\prime}$ in the sense of Radon-Nikodym in $(s, t) \backslash S$.

Proof Let $I$ be a connected component of $\Omega \backslash S$ and $[x, y] \in I \cap(s, t)$ with $\mu(\{x\})=$ $\mu(\{y\})=0$. Let $x:=x_{0}<\ldots<x_{n}:=y$ in $I \cap(s, t)$ be such that $\mu\left(\left\{x_{i}\right\}\right)=0$ and denote $h:=\max \left\{x_{i}-x_{i-1}\right\}$. By Lemma 5, $x_{i}$ is a Lebesgue point of $u$. By Proposition 2 , there exists $u_{i}$ such that (i) $u_{i}$ is monotone in $(0,1)$, (ii) $u_{i}=\tilde{u}\left(x_{i-1}\right)$ a.e. in $\left(0, x_{i-1}\right)$, (iii) $u_{i}=\tilde{u}\left(x_{i}\right)$ a.e. in $\left(x_{i}, 1\right)$ and (iv) $F\left(u_{i}\right)=\left(\tilde{u}\left(x_{i}\right)-\tilde{u}\left(x_{i-1}\right)\right)^{2} m\left(x_{i-1}, x_{i}\right)$. By Jensen inequality we have

$$
F\left(u_{i}\right) \leq \int_{\left(x_{i-1}, x_{i}\right)}\left(\frac{d u^{\prime}}{d \mu}\right)^{2} d \mu .
$$

Let $u_{h} \in \mathrm{L}^{2}(\Omega)$ be such that $u_{h}:=u$ a.e. in $\Omega \backslash\left[x_{0}, x_{n}\right]$ and $u_{h}:=u_{i}$ a.e in $\left(x_{i-1}, x_{i}\right)$. Since $u \in B V[x, y]$ we have $\lim _{h \downarrow 0}\left\|u-u_{h}\right\|_{\mathrm{L}^{2}(\Omega)}=0$. Then we deduce from Proposition 3 and Eq. 3.27 that

$$
\begin{aligned}
\mu_{u}((x, y))=F(u,(x, y)) & \leq \liminf _{h \downarrow 0} F\left(u_{h},(x, y)\right) \\
& \leq \liminf _{h \downarrow 0} \sum_{i=1}^{n} F\left(u_{h},\left[x_{i-1}, x_{i}\right]\right) \\
& \leq \liminf _{h \downarrow 0} \sum_{i=1}^{n} F\left(u_{i},\left[x_{i-1}, x_{i}\right]\right) \\
& \leq \liminf _{h \downarrow 0} \sum_{i=1}^{n} F\left(u_{i}\right) \\
& \leq \int_{(x, y)}\left(\frac{d u^{\prime}}{d \mu}\right)^{2} d \mu
\end{aligned}
$$

Using Proposition 4, the above inequality and Lemma 6 we conclude that $F(u,(s, t)) \leq \mu_{u}((s, t)) \leq \mu_{u}((s, t) \backslash S) \leq \int_{(s, t) \backslash S}\left(\frac{d u^{\prime}}{d \mu}\right)^{2} d \mu \leq F(u,(s, t))$.

Proof of existence of $(S, \mu)$ in Theorem 1 is complete. Uniqueness is ensured by the fact that, when $F$ is a functional represented as in Eq. 3.4, then $S$ has to satisfy Eq. 3.13 and $\mu$ has to satisfy the condition of Proposition 5. 


\subsection{Proof of the Closure Result}

\subsubsection{Locality is a Closed Notion}

Proposition 6 The set $\mathcal{L}$ is closed.

Proof Let $\left(F_{n}\right)$ be a sequence in $\mathcal{L}$ and $F \in \mathcal{Q}$. Assume that, for any sequence $\left(u_{n}\right)$ converging strongly in $\mathrm{L}^{2}(\Omega)$ to some $u$, $\liminf _{n} F_{n}\left(u_{n}\right) \geq F(u)$. Assume also that for any $u \in \mathrm{L}^{2}(\Omega)$ there exists $\left(u_{n}\right)$ converging strongly to $u$ and such that $\lim \sup _{n} F_{n}\left(u_{n}\right) \leq F(u)$.

Let $u \in \mathrm{L}^{2}(\Omega)$ and $x$ be a Lebesgue point of $u$. There exists a sequence $\left(u_{n}\right)$ in $\mathrm{L}^{2}(\Omega)$ such that $\lim _{n}\left\|u-u_{n}\right\|_{\mathrm{L}^{2}(\Omega)}=0, \lim _{n} F_{n}\left(u_{n}\right)=F(u)$ and such that $\left(u_{n}\right)$ converges to $u$ almost everywhere in $\Omega$. Choose two sequences $\left(s_{p}\right)$ and $\left(t_{p}\right)$ in $\Omega$, such that $\left(s_{p}\right)$ increases to $x,\left(t_{p}\right)$ decreases to $x, s_{p}$ and $t_{p}$ are Lebesgue points of $u$ and of all $u_{n}$ and such that $\lim _{n} \tilde{u}_{n}\left(s_{p}\right)=\tilde{u}\left(s_{p}\right), \lim _{n} \tilde{u}_{n}\left(t_{p}\right)=\tilde{u}\left(t_{p}\right)$ and $\lim _{p} \tilde{u}\left(s_{p}\right)=$ $\lim _{p} \tilde{u}\left(t_{p}\right)=\tilde{u}(x)$. As $F_{n}$ belongs to $\mathcal{L}$, we have by Proposition 1

$$
F_{n}\left(u_{n}\right)=F_{n}\left({\overline{\left(u_{n}\right)_{s_{p}}^{\ell}}}^{\ell}\right)+F_{n}\left({\overline{\left(u_{n}\right)_{t_{p}}^{\ell}}}_{-}-{\overline{\left(u_{n}\right)_{s_{p}}}}_{\ell}\right)+F_{n}\left({\overline{\left(u_{n}\right)_{t_{p}}^{r}}}^{r} .\right.
$$

The choice we made for the sequences $\left(s_{p}\right)$ and $\left(t_{p}\right)$ ensures that $\overline{\left(u_{n}\right)_{s_{p}}^{\ell}}$ converges strongly to $\overline{(u)}_{s_{p}}^{\ell}$, as $n$ tends to infinity and that ${\overline{(u)_{s_{p}}}}_{s_{p}}$ converges strongly to $\overline{(u)}_{x}^{\ell}$, as $p$ tends to infinity. The same holds for ${\overline{\left(u_{n}\right)_{t_{p}}^{r}}}_{\text {. Then }}$

$$
\begin{aligned}
F(u) & \geq \liminf _{p}\left(\liminf _{n} F_{n}\left(\overline{\left(u_{n}\right)_{s_{p}}^{\ell}}\right)+\liminf _{n} F_{n}\left(\overline{\left(u_{n}\right)_{t_{p}}^{r}}\right)\right) \\
& \geq \liminf _{p} F\left(\bar{u}_{s_{p}}^{\ell}\right)+\liminf _{p} F\left(\bar{u}_{t_{p}}^{r}\right) \\
& \geq F\left(\bar{u}_{x}^{\ell}\right)+F\left(\bar{u}_{x}^{r}\right) .
\end{aligned}
$$

On the other hand, there exist $\left(v_{n}\right)$ and $\left(w_{n}\right)$ such that $\lim _{n}\left\|\bar{u}_{x}^{\ell}-v_{n}\right\|_{\mathrm{L}^{2}(\Omega)}=0$, $\lim _{n} F_{n}\left(v_{n}\right)=F\left(\bar{u}_{x}^{\ell}\right)$ and $\left(v_{n}\right)$ converges to $\bar{u}_{x}^{\ell}$ almost everywhere in $\Omega, \lim _{n} \| \bar{u}_{x}^{r}-$ $w_{n} \|_{\mathrm{L}^{2}(\Omega)}=0, \lim _{n} F_{n}\left(w_{n}\right)=F\left(\bar{u}_{x}^{r}\right)$ and $\left(w_{n}\right)$ converges to $\bar{u}_{x}^{r}$ almost everywhere in $\Omega$.

Choose two sequences $\left(s_{p}\right)$ and $\left(t_{p}\right)$ such that $\left(s_{p}\right)$ increases to $x,\left(t_{p}\right)$ decreases to $x$, all $s_{p}$ and $t_{p}$ are Lebesgue points of $u, v_{n}$ and $w_{n}, \lim _{n} \tilde{v}_{n}\left(s_{p}\right)=\tilde{u}\left(s_{p}\right), \lim _{n} \tilde{w}_{n}\left(t_{p}\right)=$ $\tilde{u}\left(t_{p}\right)$ and $\lim _{p} \tilde{u}\left(s_{p}\right)=\lim _{p} \tilde{u}\left(t_{p}\right)=\tilde{u}(x)$. We have

$$
\begin{aligned}
F\left(\bar{u}_{x}^{\ell}\right)+F\left(\bar{u}_{x}^{r}\right) & =\lim _{n}\left(F_{n}\left(v_{n}\right)+F_{n}\left(w_{n}\right)\right) \\
& \geq \liminf _{p}\left(\liminf _{n}\left(F_{n}\left(\overline{\left(v_{n}\right)_{s_{p}}^{\ell}}\right)\right)+F_{n}\left(\overline{\left(w_{n}\right)_{t_{p}}^{r}}\right)\right) \\
& \geq \liminf _{p}\left(\liminf _{n} F_{n}\left(\overline{\left(v_{n}\right)_{s_{p}}^{\ell}}+\overline{\left(w_{n}\right)_{t_{p}}^{r}}\right)\right) \\
& \geq \liminf _{p} F\left(\bar{u}_{s_{p}}^{\ell}+\bar{u}_{t_{p}}^{r}\right) \\
& \geq F\left(\bar{u}_{x}^{\ell}+\bar{u}_{x}^{r}\right) \\
& \geq F(u) .
\end{aligned}
$$

Proposition 1 then implies that $F \in \mathcal{L}$. 
Remark 7 In fact we have proved a stronger result, namely: the set $\mathcal{L}$ is closed for the $\Gamma$-convergence in the strong topology of $\mathrm{L}^{2}(\Omega)$.

\subsubsection{Proof of the Density Result}

Let $F$ be a diffusion form as defined in Eq. 2.5. It is easy to check that $u \in H^{1}(\Omega)$ if and only if $\bar{u}_{x}^{\ell} \in H^{1}(\Omega)$ and $\bar{u}_{x}^{r} \in H^{1}(\Omega)$. We then deduce from Proposition 1 that $F \in \mathcal{L}$. In this particular case, the set $S$ and the measure $\mu$ which, owing to Theorem 1 , represent $F$ are respectively the empty set and $\mu(d x)=\alpha^{-1}(x) d x$. Hence $\mathcal{D}$ is a subset of $\mathcal{L}$.

Proposition 7 Let $F \in \mathcal{L}$. Then there exists a sequence of diffusion forms $\left(F_{n}\right)$ which Mosco-converges to $F$.

Proof Let $(S, \mu)$ associated to $F$ by Theorem 1. For any integer $n$ and any $i \in$ $\{1, \ldots, n\}$, let us denote $I_{i}^{n}$ the interval $I_{i}^{n}:=\left(\frac{i-1}{n}, \frac{i}{n}\right), s_{i}^{n}:=\frac{2 i-1}{2 n}$ its center, $h_{i}^{n}$ the function defined on $(0,1)$ by

$$
h_{i}^{n}(x):= \begin{cases}n\left(x-s_{i-1}^{n}\right) & \text { if } s_{i-1}^{n}<x \leq s_{i}^{n} \\ n\left(s_{i+1}^{n}-x\right) & \text { if } s_{i}^{n}<x<s_{i+1}^{n} \\ 0 & \text { otherwise }\end{cases}
$$

and $\alpha_{i}^{n}$ the quantity defined by

$$
\frac{1}{\alpha_{i}^{n}}:= \begin{cases}n^{-1}+n \int_{\Omega \backslash S} h_{i}^{n} d \mu & \text { if }\left[\frac{i-2}{n}, \frac{i+1}{n}\right] \subset \Omega \backslash S \\ n^{3} & \text { otherwise }\end{cases}
$$

Denoting $\alpha_{n}$ the piecewise constant function $\alpha_{n}:=\sum_{i=1}^{n} \alpha_{i}^{n} 1_{I_{i}^{n}}$, we define $F_{n} \in \mathcal{D}$ by

$$
F_{n}(u):= \begin{cases}\int_{\Omega}\left(u^{\prime}(x)\right)^{2} \alpha_{n}(x) d x & \text { if } u \in H^{1}(\Omega) \\ +\infty & \text { otherwise. }\end{cases}
$$

Now let us prove that the sequence $\left(F_{n}\right)$ Mosco converges to $F$. For any $\varphi \in$ $C_{c}^{\infty}(\Omega \backslash S)$ and for $n$ large enough, we have

$$
\int_{\Omega} \frac{\varphi(x)}{\alpha_{n}(x)} d x=\frac{1}{n} \int_{\Omega \backslash S} \varphi(x) d x+\sum_{i} \int_{\Omega}\left(n \int_{I_{i}^{n}} \varphi(t) d t\right) h_{i}^{n}(x) \mu(d x) .
$$

Hence

$$
\lim _{n} \int_{\Omega} \frac{\varphi(x)}{\alpha_{n}(x)} d x=\int_{\Omega \backslash S} \varphi(x) \mu(d x)
$$

Let $\left(u_{n}\right)$ be a sequence converging to $u$ with respect to the weak topology of $\mathrm{L}^{2}(\Omega)$ and such that $\liminf _{n} F_{n}\left(u_{n}\right)=M<+\infty$. By Cauchy-Schwarz inequality, we have, for any $\varphi \in C_{c}^{\infty}(\Omega \backslash S)$,

$$
\left|\int_{\Omega} u_{n}(x) \varphi^{\prime}(x) d x\right|^{2} \leq F_{n}\left(u_{n}\right) \int_{\Omega} \frac{(\varphi(x))^{2}}{\alpha_{n}(x)} d x
$$


Then, passing to the limit as $n$ tends to infinity and using Eq. 3.28, we obtain

$$
\left|\int_{\Omega} u(x) \varphi^{\prime}(x) d x\right|^{2} \leq M \int_{\Omega \backslash S}(\varphi(x))^{2} \mu(d x) .
$$

By Theorem 1, we deduce

$$
F(u)=\int_{\Omega \backslash S}\left(\frac{d u^{\prime}}{d \mu}\right)^{2} d \mu \leq M,
$$

which proves the lower-bound inequality.

Let $u \in \mathrm{L}^{2}(\Omega)$ be such that $F(u)<+\infty$. For any $i \in\{1, \ldots, n\}$, let us denote $m_{i}^{n}:=$ $n \int_{s_{i}^{n+1}}^{s_{i+1}^{n}} u(t) d t$. We have, when $\left[\frac{i-2}{n}, \frac{i+1}{n}\right]$,

$$
\begin{aligned}
m_{i}^{n}-m_{i-1}^{n} & =n \int_{s_{i-1}^{n}}^{s_{i}^{n}}\left(u\left(t+\frac{1}{n}\right)-u(t)\right) d t=n \int_{s_{i-1}^{n}}^{s_{i}^{n}}\left(\int_{t}^{t+\frac{1}{n}} \frac{d u^{\prime}}{d \mu} d \mu\right) d t \\
& =\int_{s_{i-1}^{n}}^{s_{i+1}^{n}} h_{i}^{n} \frac{d u^{\prime}}{d \mu} d \mu=\int_{\Omega \backslash S} h_{i}^{n} \frac{d u^{\prime}}{d \mu} d \mu .
\end{aligned}
$$

Using Cauchy-Schwarz inequality, we obtain

$$
\left(m_{i}^{n}-m_{i-1}^{n}\right)^{2} \leq\left(\int_{\Omega} h_{i}^{n} d \mu\right)\left(\int_{\Omega} h_{i}^{n}\left(\frac{d u^{\prime}}{d \mu}\right)^{2} d \mu\right) .
$$

Let $u_{n}$ be the continuous function on $[0,1]$ which is affine on each $I_{i}^{n}$ and satisfies $u_{n}(0)=m_{1}^{n}, u_{n}(1)=m_{n-1}^{n}$ and $u_{n}\left(\frac{i}{n}\right)=m_{i}^{n}$ for $1 \leq i \leq n-1$. We have $\lim _{n} \| u-$ $u_{n} \|_{\mathrm{L}^{2}(\Omega)}=0$. Using Eq. 3.29, Theorem 1 and Jensen inequality, we obtain

$$
\begin{aligned}
F_{n}\left(u_{n}\right) & =\sum_{i=2}^{n-1} n \alpha_{n}\left(s_{i}\right)\left(m_{i}^{n}-m_{i-1}^{n}\right)^{2} \\
& \leq \sum_{i=2}^{n-1} \int_{\Omega \backslash S} h_{i}^{n}\left(\frac{d u^{\prime}}{d \mu}\right)^{2} d \mu+\frac{1}{n^{2}} \sum_{i=2}^{n-1}\left(m_{i}^{n}-m_{i-1}^{n}\right)^{2} \\
& \leq F(u)+\frac{4}{n} \int_{\Omega}(u(x))^{2} d x
\end{aligned}
$$

The upper-bound inequality is obtained by passing to the limit.

Proof of Theorem 2 is concluded by collecting the closure result of Proposition 7 and the density result of Proposition 7.

\section{About Locality}

Owing to Deny-Beurling formula, it is quite clear what a local regular Dirichlet form is: in the representation formula Eq. 2.8 the measure $\gamma$ should vanish.

In this section we discuss the extension of this notion of locality to non regular forms. Different criterions have been proposed in the literature which all coincide 
when applied to regular forms. We compare these different notions and we prove that none of them are preserved when passing to the limit with respect to Mosco or $\Gamma$-convergence.

\section{Definition 7}

i) We denote $\mathcal{V}$ the set of all forms $F \in \mathcal{Q}$ which satisfy: for any $\varphi$ and $\psi$ in $C_{o}^{\infty}(\mathbb{R})$ with disjoint supports and any $u \in D(F)$,

$$
F(\varphi \circ u+\psi \circ u)=F(\varphi \circ u)+F(\psi \circ u) .
$$

ii) We denote $\mathcal{S}$ the set of all forms $F \in \mathcal{Q}$ which satisfy: for any $u$ and $v$ in $\mathrm{L}^{2}(\Omega)$ with disjoint supports,

$$
F(u+v)=F(u)+F(v) .
$$

iii) We denote $\mathcal{M}$ the set of all forms $F \in \mathcal{Q}$ which satisfy: there exists a mapping $\mu$ which associates to any $u \in \mathrm{L}^{2}(\Omega)$ a Borel measure $\mu_{u}$ such that

$$
F(u)=\mu_{u}(\Omega) \text { and } \mu_{u}(B)=\mu_{v}(B)
$$

whenever $u$ and $v$ coincide on an open set $O$ containing the Borel set $B$.

iv) We denote $\mathcal{R}$ the set of all forms $F \in \mathcal{Q}$ which satisfy: there exists a mapping $\mu$ which associates to any $u \in \mathrm{L}^{2}(\Omega)$ a Borel measure $\mu_{u}$ such that, for every open interval $I \subset \Omega$,

$$
\mu_{u}(I)=F(u, I)
$$

where $F(u, I)$ is defined by Eqs. 3.2-3.3.

Definition (i) can be found in [5] while definition (ii) can be found in [1] or [13]. There are closely related : indeed any $u$ defines a new quadratic and Markovian form $\varphi \mapsto F(\varphi \circ u)$. Definition (i) means that these new forms satisfy definition (ii). Roughly speaking, definition (ii) forbids interactions between distant zones: indeed as the supports of $u$ and $v$ are closed, assuming that there are disjoint means that their distance is positive.

Definitions (iii) or (iv) like our Definition 6 associate to any function $u \in \mathrm{L}^{2}(\Omega)$ an energy density (a measure) $\mu_{u}$. Note that this measure is not necessarily finite on compact sets. Definition (iii) simply asks that the measure $\mu_{u}$ depends in a "local way" on $u$. The mapping $\mu$ corresponds to the functional introduced a priori in definition 15.21 of [12] and the property, $\mu_{u}(B)=\mu_{v}(B)$ whenever $u$ and $v$ coincide on an open set $O \subset B$, is precisely the characterization of locality given in [12]. Definition (iv) is more precise: it asks $\mu_{u}$ to coincide on every open interval $I$ to the relaxed energy on $I$. In our definition 6 we ask, in addition, that $\mu_{u}$ does not concentrate on the Lebesgue points of $u$.

The relations between these different notions of locality are made precise by the following proposition.

\section{Proposition 8}

i) We have the following strict inclusions

$$
\mathcal{D} \subsetneq \mathcal{L} \subsetneq \mathcal{R} \subsetneq \mathcal{M} \subsetneq \mathcal{S} \text { and } \mathcal{D} \subsetneq \mathcal{V} \subsetneq \mathcal{S} .
$$

ii) $\mathcal{R}, \mathcal{M}, \mathcal{V}$ and $\mathcal{S}$ are not closed with respect to the Mosco convergence. 
All inclusions in point (i) are almost direct consequences of definitions. To check that these inclusions are strict and to prove (ii) it is enough to consider suitable examples.

In Example 1 we exhibited a functional $F$ which is the limit of a sequence of functionals in $\mathcal{D}$. It belongs to $\mathcal{L}=\overline{\mathcal{D}}$. We let the reader check that it does not belong to $\mathcal{V}$. This example proves that $\mathcal{L}$ is not included in $\mathcal{V}$ and that $\mathcal{V}$ is not closed.

The theory, established in the case of functionals $G$ with dense domain [5], states the existence of a measure $\sigma^{u}$ satisfying $\left.G(\varphi \circ u)=\int_{\mathbb{R}} \varphi^{\prime}(t)\right)^{2} \sigma^{u}(d t)$ for every $\varphi \in$ $C_{c}^{1}(\mathbb{R})$. Here it cannot be applied to $F$ (the domain of $F$ is not dense in $\mathrm{L}^{2}(\Omega)$ !). Moreover, one can directly check that $F$ cannot be represented in this way.

Proving the other assertions needs more sophisticated examples. To construct these examples we need the following lemma

Lemma 8 Let $n \in \mathbb{N}$ and let $\left\{\alpha_{i}\right\}_{1 \leq i \leq n}$ be a family of positive reals with $\sum_{i=1}^{n} \alpha_{i}=1$. Then there exists a partition $\left\{A_{i}\right\}_{1 \leq i \leq n}$ of $[0,1]$ made by Borel sets $A_{i}$ satisfying $\left|A_{i}\right|=$ $\alpha_{i}$ and $\left|A_{i} \cap I\right|>0$ for every $i$ and every non empty open interval $I \subset[0,1]$.

For lack of space, we skip the proof of this lemma which is obtained by an induction argument. When $n=2$ a construction analogous to the classical construction of Cantor set is used. In the following we refer to partitions satisfying Lemma 8 as essential partitions.

Example 2 Let $A \subset \Omega$ and $G_{A}$ be the functional defined by

$$
G_{A}(u)= \begin{cases}0 & \text { if } u=a 1_{A}+b 1_{\Omega \backslash A} \text { for some } a, b \in \mathbb{R}, \\ +\infty & \text { otherwise. }\end{cases}
$$

We let the reader check that, when $\{A, \Omega \backslash A\}$ is an essential partition, the functional $G_{A}$ belongs to $\mathcal{R} \backslash \mathcal{L}$.

Example 3 Let $\left\{A_{n}, \Omega \backslash A_{n}\right\}$ be a sequence of essential partitions satisfying $\left|A_{n}\right|=$ $(n+1)^{-1}$. Let us define

$$
B_{n}:=\frac{1}{3} A_{1} \cup\left(\frac{1}{3}+\frac{1}{3} A_{n}\right) \cup\left(\frac{2}{3}+\frac{1}{3} A_{1}\right)
$$

and

$$
B_{\infty}:=\frac{1}{3} A_{1} \cup\left(\frac{2}{3}+\frac{1}{3} A_{1}\right)
$$

Note that $\left\{B_{n}, \Omega \backslash B_{n}\right\}$ is still an essential partition while it is not the case for $\left\{B_{\infty}, \Omega \backslash B_{\infty}\right\}$. We let the reader check that i) the sequence of functionals $G_{B_{n}}$ Mosco converges to $G_{B_{\infty}}$ ii) the limit functional $G_{B_{\infty}}$ does not belong to $\mathcal{S}$. This example proves that neither $\mathcal{R}$, nor $\mathcal{M}$, nor $\mathcal{S}$ are closed.

Example 4 Let $\{A, \Omega \backslash A\}$ be an essential partition. Define $F$ by

$$
F(u):=\int_{\Omega}\left(\left(v^{\prime}(x)\right)^{2}+\left(w^{\prime}(x)\right)^{2}+(v(x)-w(x))^{2}\right) d x,
$$


if $u=v 1_{A}+w 1_{\Omega \backslash A}$ a.e. in $\Omega$, for some $v, w$ in $H^{1}(\Omega)$ and $F(u):=+\infty$ otherwise. We let the reader check that $F$ belongs to $\mathcal{M} \backslash \mathcal{R}$.

Example 5 Let us consider an essential partition $\left\{A_{1}, A_{2}, A_{3}\right\}$ and the functional $F$ defined by

$$
F(u):=\left\{\begin{array}{cc}
\int_{\left(0, \frac{1}{3}\right) \times\left(\frac{2}{3}, 1\right)}\left(u_{2}(x)-u_{2}(y)\right)^{2} d x d y, & \text { if } u=u_{1} 1_{A_{1}}+u_{2} 1_{A_{2}}+u_{3} 1_{A_{3}}, \\
& u_{1} \in \mathbb{R}, u_{2} \in \mathrm{L}^{2}(\Omega), u_{3} \in \mathbb{R}, \\
\text { and }\left(u_{1} \leq u_{2} \leq u_{3} \text { or } u_{3} \leq u_{2} \leq u_{1}\right), \\
+\infty, & \text { otherwise. }
\end{array}\right.
$$

We let the reader check that $F$ belongs to $\mathcal{S} \backslash \mathcal{M}$.

\section{References}

1. Applebaum, D.: Levy Processes and Stochastic Calculus. Cambridge University Press, Cambridge (2004)

2. Bellieud, M., Bouchitté, G.: Homogénéisation en présence de fibres de grandes conductivité. C.R. Acad. Sci. Paris Sér I Math. 323, 1135-1140 (1996)

3. Bellieud, M., Bouchitté, G.: Homogenization of elliptic problems in a fiber reinforced structure. Non local effects. Ann. Scuola Norm. Sup. Pisa Cl. Sci. 26, 407-436 (1998)

4. Beurling, A., Deny, J.: Dirichlet spaces. Proc. Natl. Acad. Sci. U.S.A. 45, 208-215 (1959)

5. Bouleau, H.F.: Dirichlet forms and analysis on Wiener space. de Gruyter Studies in Mathematics, vol. 14. de Gruyter, Berlin (1991)

6. Briane, M.: Non-local effects in two-dimensional conductivity. Arch. Ration. Mech. Anal. 182(2), 255-267 (2006)

7. Briane, M., Tchou, N.: Fibered microstructures for some nonlocal Dirichlet forms. Ann. Scuola Norm. Sup. Pisa Cl. Sci. 4, 30(3-4), 681-711 (2001)

8. Buttazzo, G., Dal Maso, G.: Г-limits of integral functionals. J. Anal. Math. 37, 145-185 (1980)

9. Camar-Eddine, M.: Closure of the set of diffusion functionals and that of elasticity with respect to the Mosco-convergence. Thesis of Toulon University, France (2002)

10. Camar-Eddine, M., Seppecher, P.: Non-local interactions resulting from the homogenization of a linear diffusive medium. C. R. Acad. Sci. Paris Ser. I Math. 332, 485-490 (2001)

11. Camar-Eddine, M., Seppecher, P.: Closure of the set of diffusion functionals with respect to the Mosco-convergence. Math. Models Methods Appl. Sci. 12(8), 1153-1176 (2002)

12. Dal Maso, G.: An introduction to $\Gamma$-convergence. Progress in Linear Diff. Eq. and Their App. Birkhäuser, Boston (1993)

13. Fukushima, M.: Dirichlet Forms and Markov Processes. North Holland, Amsterdam (1980)

14. Hashin, Z., Shtrikman, S.: A variational approach to the theory of the elastic behavior of multiphase materials. J. Mech. Phys. Solids 11, 127-140 (1963)

15. Milton, G.W.: Composite materials with Poisson's ratios close to -1. J. Mech. Phys. Solids 40(5), 1105-1137 (1992)

16. Mosco, U.: Composite media and asymptotic Dirichlet forms. J. Funct. Anal. 123, 368-421 (1994)

17. Murat, F.: A strange term brought from somewhere else. Nonlinear Differential Equations And Their Applications. Collège de France Seminar, vol. II, pp. 98-138, 389-390, (Paris, 1979/1980), Res. Notes in Math., 60. Pitman, Boston (1982)

18. Murat, F., Tartar L.: Calculus of variations and homogenization. Topics in the mathematical modelling of composite materials. Progr. Nonlinear Differential Equations Appl., vol. 31, pp. 139-173. Birkhauser Boston, Boston (1997)

19. Tartar, L.: Estimations de coefficients homogénéisés. In: Glowinski, R., Lions, J.L. (eds.) Computing Methods in Applied Sciences And Engineering. Lecture Notes in Math., vol. 704, pp. 364-373. Springer, Berlin Heidelberg New York (1978) 